\title{
Wideband Interference Mitigation for Synthetic Aperture Radar Data Based on Adaptive Compressed Sampling
}

\author{
Guojing Li ${ }^{1,2, a}$, Guozhu Liu ${ }^{1}$, Wei Ye ${ }^{2}$, Shuya Kong ${ }^{2}$ and Yuan Bo ${ }^{2}$ \\ ${ }^{1}$ State Key Laboratory of Complex Electromagnetic Environment Effects on Electronics and \\ Information System, Luoyang, 471003, China \\ ${ }^{2}$ Equipment Academy, Beijing, 101416, China \\ aleeguojing1014@163.com
}

Keywords: Synthetic aperture radar; wideband interference mitigation; compressed sensing; adaptive measurement; projection filtering

\begin{abstract}
Wideband interference (WBI) is a threat to synthetic aperture radar because it occupies a large bandwidth and lead to a poor quality of SAR images. This paper presents a method of wideband interference mitigation for SAR imaging based on adaptive compressed sampling. First, an adaptive and compressed measurement, projection filter banks and an interference detector are constructed. Based on minimum output energy criterion, WBI is extracted through joint detection after projected by filter banks and then mitigated through adaptively adjusting the compressed measurement 'away' from the interference positions. The experimental results of the simulated data indicate that the method proposed is effective.
\end{abstract}

\section{Introduction}

Synthetic aperture radar (SAR) plays a significant role in the field of civil exploration and military reconnaissance owing to its capability of all-time, all-weather and high-resolution imaging, and for this reason many researchers have been always exploring new SAR imaging methods to improve the performance of super-resolution and anti-interference in recent years. However, the increasing requirement for high-resolution imaging means that the radar system must be able to receive and process huge amounts of data in time, which is hindered by expensive devices. Besides, the shortage of spectrum resources poses a potential threat to the security of SAR system with a large amount of natural and man-made interference emerging. Therefore, it is urgent to explore new imaging method from the aspect of signal or data processing to deal with these problems.

The theory of compressed sensing (CS) [1] is widely applied in SAR imaging recently, making it possible to represent the scene of interest with the minimal amount of observations [2]. For the purpose of super-resolution, the application of CS theory can improve the quality of SAR images from a few measurements through making the most of the sparsity instead of matched filtering and fits for different forms of signal such as ultra-wideband (UWB) [3], random frequency [4], etc.

However, the existence of noise and interference might destroy the structure of sparse information and then lead to a deterioration of the signal reconstruction. Wideband interference (WBI), especially WBI with noise modulation, is more destructive to SAR imaging, since it occupies a wideband spectrum and thus reduce the signal-to-noise. The combination of traditional methods including transform domain notching, Eigen-subspace filtering, and independent component analysis convert a wideband spectrum to series of narrowband ones and then suppress the interference components in turn [5],[6], which is operated with a large amount of calculation and ineffective on WBI with noise modulation.

In this paper, we propose a method of wideband interference mitigation for SAR data based on adaptive compressed sensing. WBI is extracted through joint detection after projected by filter banks and then mitigated through adjusting the compressed measurement adaptively 'away' from the interference positions. It should be noted that the WBI mentioned below refer to the wideband noise interference. 


\section{Compressed synthetic aperture radar imaging}

In the standard compressed sensing framework, the data is usually acquired via the linear measurements $\boldsymbol{y}=\boldsymbol{\Phi} \boldsymbol{x}$, where $\boldsymbol{\Phi}$ is an $M \times N$ measurement matrix and $y \in \mathrm{R}^{M}$ is the vector of samples acquired. $\boldsymbol{x}$ is sparse on a $N$-dimensional space with $\Psi=\left[\Psi_{1}, \Psi_{2}, \ldots, \Psi_{N}\right]$ as the sparsity basis providing a $K$-sparse representation $\boldsymbol{x}=\boldsymbol{\Psi} \boldsymbol{\alpha}$, where $\boldsymbol{\alpha}=\left[\alpha_{1}, \alpha_{2}, \ldots, \alpha_{N}\right]$ are the associated coefficients. The basic recovery approach is to search for the sparsest vector $\boldsymbol{x}$ which is consistent with the measurement $y$, leading to solving the $\ell_{0}$-miminization problem, i.e.

$\min \|\boldsymbol{x}\|_{0} \quad$ subject to $\quad \boldsymbol{y}=\boldsymbol{\Phi} \boldsymbol{x}$,

which is, however, computationally intractable as a NP-hard combinatorial minimization problem. Often we substitute $\ell_{0}$-miminization with $\ell_{1}$-miminization or matching pursuit.

Assume that the SAR data $\boldsymbol{x}_{N \times 1}$ is sensed by a compressed matrix $\boldsymbol{\Phi}_{M \times N}$, then the sparse dictionary $\boldsymbol{\Psi}_{N \times N}$ is made of reference data $\hat{\boldsymbol{x}}_{N \times 1}$ with delay and the recovery matrix $\boldsymbol{\Theta}_{M \times N}$ is obtained by the product of $\boldsymbol{\Phi}_{M \times N}$ and $\boldsymbol{\Psi}_{N \times N}$. We employ the orthogonal matching pursuit (OMP) algorithm to reconstruct the image by obtaining sparse coefficient $\boldsymbol{\alpha}_{N \times 1}$.

\section{WBI mitigation based on adaptive compressed sampling}

Suppose that contaminated SAR data $\boldsymbol{x} \in R^{N}$ consists of two components, i.e.

$$
x=x_{S}+x_{I},
$$

where $\boldsymbol{x}_{S}$ is the data of interest and $\boldsymbol{x}_{I}$ represents the WBI. The aim of interference component mitigation is to remove the contribution of $\boldsymbol{x}_{I}$ from the measurements $\boldsymbol{y}$ while preserving the information of $\boldsymbol{x}_{S}$. The interference mitigation is conducted by a linear operator $\boldsymbol{P}$ solely based on the sensing matrix $\boldsymbol{\Phi}$ and the support of $\boldsymbol{x}_{I}$, i.e.

$$
\boldsymbol{P} \boldsymbol{y}=\boldsymbol{P} \Phi \boldsymbol{x}_{S}+\boldsymbol{P} \Phi \boldsymbol{x}_{I}=\boldsymbol{P} \Phi \boldsymbol{x}_{S},
$$

where the matrix $\boldsymbol{P} \boldsymbol{\Phi} \boldsymbol{x}_{S}$ satisfies the RIP. However, the formula above-mentioned depends heavily on sparse prior information which is hardly possible to estimate from WBI.

Our scheme of WBI mitigation avoids the impact of WBI from the source as much as possible rather than extracting and mitigating interference from the contaminated measurement. The compressed measurement contains information of both signal and interference since their sparse bases are not orthogonal. We set a cost function with the output projected by a filtering operator $\boldsymbol{P}$ with the criterion of minimum energy [7], i.e.

$$
E\left[\left\langle\boldsymbol{P}_{i}, \boldsymbol{y}\right\rangle^{2}\right]=\boldsymbol{P}_{i}^{H} \boldsymbol{\Phi} \mathbf{R}_{\alpha \alpha} \boldsymbol{\Phi}^{H} \boldsymbol{P}_{i},
$$

where $\boldsymbol{R}_{\alpha \alpha}$ denotes the auto-correlation matrix of the sparse coefficient, and $\boldsymbol{P}_{i}$ is the operator for the ith column of the measurement. The optimal projection operator is obtained through Lagrange method, i.e.

$$
\boldsymbol{P}_{i, o p t}=\left(\boldsymbol{\Phi} \mathbf{R}_{\alpha \alpha} \boldsymbol{\Phi}^{H}\right)^{-1} \boldsymbol{\theta}_{i}\left[\boldsymbol{\theta}_{i}^{H}\left(\mathbf{\Phi} \mathbf{R}_{\alpha \alpha} \boldsymbol{\Phi}^{H}\right)^{-1} \boldsymbol{\theta}_{i}\right]^{-1},
$$

where $\boldsymbol{\theta}_{i}$ is the $i$ th column vector of the recovery matrix $\boldsymbol{\Theta}$. And then the minimum energy of the output is

$$
E\left[\left\langle\boldsymbol{P}_{i, o p t}, \boldsymbol{y}\right\rangle^{2}\right]=\left(\boldsymbol{\theta}_{i}^{-1}\left(\boldsymbol{\Phi} \boldsymbol{R}_{\alpha \alpha} \boldsymbol{\Phi}^{H}\right)^{-1} \boldsymbol{\theta}_{i}\right)^{-1} .
$$

The output projected is utilized by a joint detector to extract the position information of the WBI which is transferred to the compressed measurement front-end as a feedback factor. The sensing matrix is adjusted adaptively through setting the column vector corresponding to the interference 
position information to zero. If there is no interference component or the interference energy is below a certain threshold, the SAR image is reconstructed with OMP algorithm.

\section{Experimental simulation results}

Experimental simulations are conducted using a simulated Strip-map SAR data in contaminated with wideband noise interference. We set five point targets of random positions and amplitudes in an imaging scene with the size of $400 \mathrm{~m} \times 300 \mathrm{~m}$ (Azimuth-Range). A set of WBI are added with different values of power. We utilize interference-to-signal ratio (ISR) to calculate the interference level (dB) and define compressed ratio (CR) as the sampling proportion of raw data. In our experiments, we set CR to 0.5 and meanwhile set ISR to $22 \mathrm{~dB}$.

The targets are hardly distinguished from the background interference in the azimuth-range SAR image shown in Fig.1(a) without any interference mitigation processing, and after processing with the approach we proposed, the targets are clearly visible in Fig.1(b). By comparing with the two figures, our approach to mitigate WBI and improve the quality of SAR imaging has been validated preliminarily.

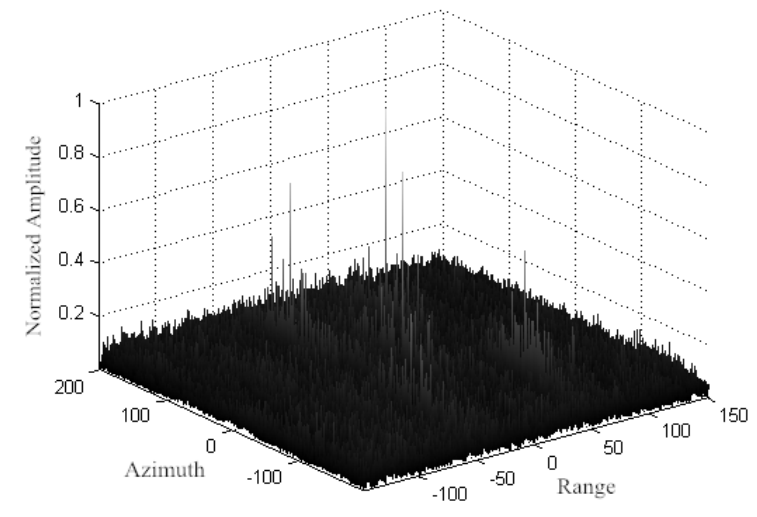

(a) without WBI mitigation

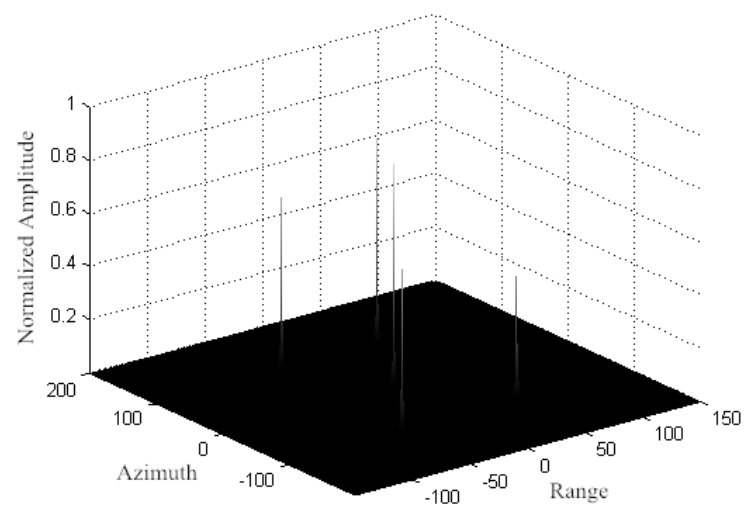

(b) with WBI mitigation

Fig.1 The range-azimuth imaging results

In order to check the performance of our approach objectively, we employ interference mitigation ratio expressed by $\gamma$ as an indicator for evaluation, reflecting the level reduction of interference energy after mitigation, i.e.

$$
\gamma=10 \lg \left(\frac{\sum_{n}|x(n)|^{2}}{\sum_{n}|\hat{x}(n)|^{2}}\right),
$$

where $x(n)$ is the contaminated data, $\hat{x}(n)$ is the recovered data after interference mitigating, and $n$ denotes the number of samples processed. Meanwhile, we employ data distortion expressed by $\mu$ as an indicator representing the impairment of data, i.e.

$$
\mu=10 \lg \left(\frac{\sum_{n}\left|x_{0}(n)-\hat{x}(n)\right|^{2}}{\sum_{n}\left|x_{0}(n)\right|^{2}}\right),
$$

where $x_{0}(n)$ is the clean data. These two indicators above evaluate the performance from different perspective and neither is dispensable.

For the purpose of checking the mitigation performance with indicators aforementioned, we carry out simulations with ISR ranging from $0 \mathrm{~dB}$ to $30 \mathrm{~dB}$. As is shown in Fig.2, each of the performance curves presents a smooth fluctuation when ISR $<25 \mathrm{~dB}$, yet a sharp deterioration when ISR $>25 \mathrm{~dB}$. 


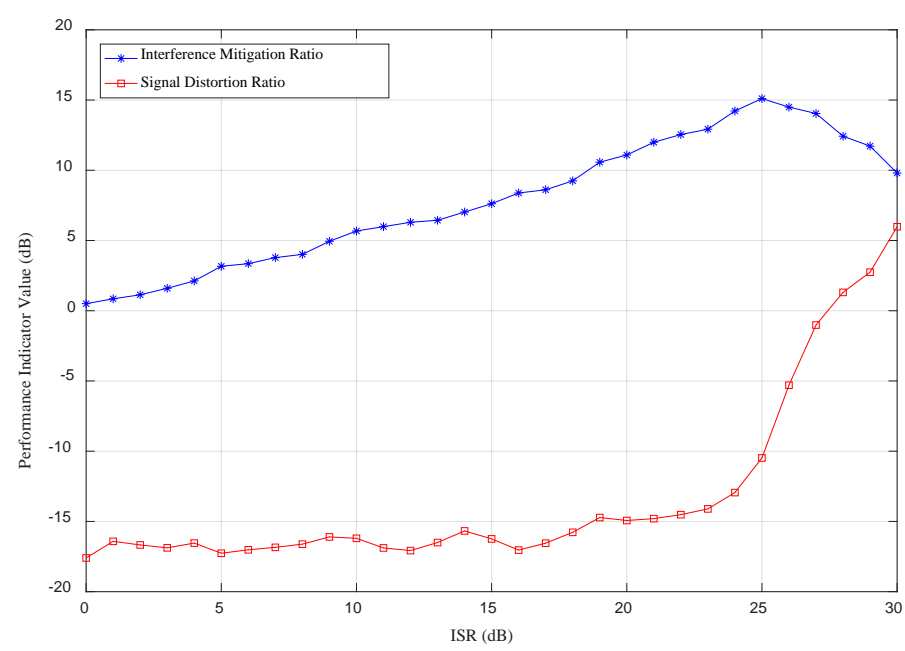

Fig.2 Interference mitigation performace with different level of ISR

Now we give the explanation and analysis about why this phenomenon occurs. Once the energy of interference is greater, the positional information of interference might be misjudged during the period of joint detection, leading to a result that adaptive measurement would mistake the subspace of interference for data of interest. Therefore, how to improve the accuracy of detection and reduce the probability of misjudgment when encountered with strong interference is one aspect of our follow-up work.

\section{Conclusions}

We present an effective approach of wideband interference mitigation for synthetic aperture radar based on an adaptive measurement framework including subspace projection and interference joint detection in compressed domain. The experimental results with contaminated simulation data of SAR confirm the validity of our approach. In the future, we would like to develop more intelligent algorithms to reduce adaptive measurement error with machine learning, which would further improve the effectiveness of interference mitigation.

\section{References}

[1] D. L. Donoho, in: IEEE Transactions on Information Theory. Vol. 52(2006), no.4, p. 1289-1306

[2] M. Cetin, I. Stojanovic, O. Onhon, et al, in: IEEE Signal Processing Magazine. Vol. 31(2014), no. 4, p. 27-40

[3] L. H. Nguyen, T. Tran and T. Do, in: IEEE Transactions on Aerospace and Electronic Systems, Vol. 50(2014), no. 2, p. 940-958

[4] J. Yang, J. Thompson, X. Huang, et al, in: IEEE Transactions on Geoscience and Remote Sensing. Vol. 51(2013), no. 2, p. 983-994

[5] M. Tao, F. Zhou, Z. Zhang, in: IEEE Transactions on Geoscience and Remote Sensing. Vol. 54(2016), no. 1, p. 74-87

[6] S. Zhang, M. Xing, R. Guo, et al, in: IEEE Transactions on Geoscience and Remote Sensing. Vol. 49(2011), no. 10, p. 3765-3779

[7] R. Kang, P. Wu, H. Yu, in: Acta Physica Sinica. Vol. 63(2014), no. 20, p. 200701-200701 (in Chinese) 